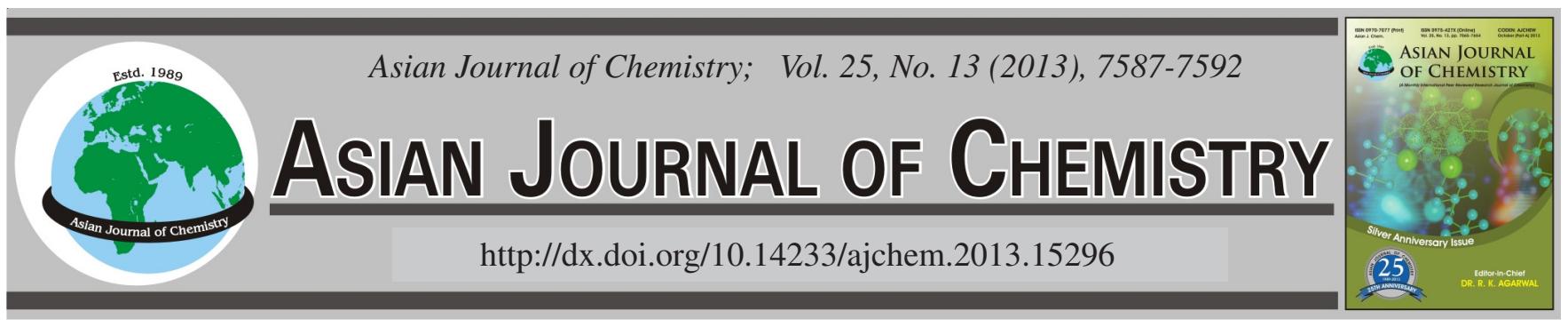

\title{
Study on Discriminating Flue-Cured Tobacco by Volatile Compounds Related to Geographical Origin and Cultivar
}

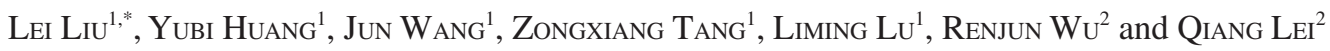

${ }^{1}$ College of Agronomy, Sichuan Agricultural University, Chengdu 611130, Sichuan Province, P.R. China

${ }^{2}$ Science and Technology Department of Sichuan Tobacco Company, P.R. China

*Corresponding author: Fax: +96 28 86290870; Tel: +86 28 86290970; E-mail: srars@126.com

(Received: 6 February 2013;

Accepted: 1 July 2013)

AJC-13758

\begin{abstract}
To distinguish the geographical origins and cultivars of flue-cured tobacco, 35 samples cultivated in three districts (Liangshan, Yibin and Gunagyuan) of Sichuan Province (southwest of China) were collected. Volatile compounds were analyzed with gas chromatography/mass spectrometry (GC/MS) and 56 different volatile compounds were tentatively identified and semi-quantified. The data was subjected to SIMCA-P+13.0 Software. Principal component analysis (PCA) and partial least squares discrimination analysis (PLS-DA) were used to predict the tobacco's geographical origin or cultivar using soft independent modeling of class analogy (SIMCA) statistical techniques. Both PCA and PLS-DA indicate that all samples could be classified into three clusters coincident to the three geographical origins. The PLS-DA have an average recognition ability of $91.4 \%$. However, analysis on discriminating tobacco cultivars by volatiles revealed that it is difficult to find any volatile as a distinctive mark to identify these six tobacco cultivars cultured in Sichuan Province.
\end{abstract}

Key Words: Classification, Tobacco, Cultivar, Volatile, Geographical origin.

\section{INTRODUCTION}

Characteristics of agro-product quality have long been linked with their geographical origin. Identification of the geographical origin of agro-products is effective in protecting the quality and safety of agro-product ${ }^{1}$. Increased demand for high-quality and authentic agro-products has given rise to the need to identify and trace the geographical origin of agroproducts. Chemical composition and content of agro-products are dominant factors for product quality and can serve as geographical indications of origin. Many modern analytical instruments used in analyzing mineral content $\mathrm{t}^{2-4}$, isotope content and ratio ${ }^{5,6}$, DNA analysis ${ }^{7,8}$ and volatile compounds ${ }^{9,10}$, the collection of numerous data about the chemical characteristics of agro-products is possible. Furthermore, when combined with chemometrics, the geographical origin of agro-products can be discerned ${ }^{11}$.

It is generally assumed that plant volatiles could attract pollinators $^{12}$, increase tolerance ${ }^{13}$, serve as plant defenses ${ }^{14,15}$ and also act in plant-plant communication. However, characterizing the composition and content of volatiles in the same kind of agro-products could be very diverse, due to the differences of many influencing factors such as variety, processing technology, environmental factors, etc. Examination of the volatiles might be considered as a strategy enabling products' authentication since composition is known to vary widely with multiple factors involved in agro-production. For example, depending on the difference in volatiles, milk samples were classified correctly into groups which were consistent with the type of forage eaten by the cattle ${ }^{16}$. Characterization of volatiles was also highly suitable for varietal discrimination of hops (Humulus lupulus L.) $)^{17}$ or wines according to varieties ${ }^{18}$. Environmental factors such as light, temperature and moisture status can greatly affect the emission of volatiles, including the yield and composition of essential oils ${ }^{19,20}$. Studies also show that geographical origins of coffee beans ${ }^{21}$, honeys ${ }^{22,23}$, olive oils ${ }^{24}$, green teas ${ }^{25}$, cheese $^{26}$ and many other agro-products could be discriminated based on the diversity of volatiles.

As far as flavour and taste are concerned, volatiles are one of the most important influencing factors, especially for tobacco. Many volatile compounds in tobacco can be transferred from cut tobacco leaf to smoke by volatilization without any structural change.

Flue-cured tobacco (Nicotiana tabacum L.) is one of the most important commercial crops. The major volatile compounds reported in flue-cured tobacco are neophytadiene, aromatic ketones, aromatic alcohols, aliphatic acids and aromatic aldehydes. These compounds usually comprise more than $90 \%$ of the total volatile compounds and contribute to the aroma of flue-cured tobacco ${ }^{27}$. Volatile compounds in 
tobacco are affected widely and deeply by many factors related to growing area ${ }^{28-30}$. It is interesting to clarify whether the geographical origin of tobacco could be discriminated or traced based on the difference of volatiles and whether geographical indications of volatiles could be taken as a quality-mark of flue-cured tobacco.

However, few efforts have been made to identify tobacco's geographical origin and cultivar by analyzing volatile compounds. Furthermore, no information exists in the literature on classification of flue-cured tobacco planted in Sichuan Province. In this study we are trying to discriminate the geographical origin and cultivars of flue-cured tobacco planted in Sichuan Province according to volatile compounds.

\section{EXPERIMENTAL}

Tobacco samples: Tobacco leaves were harvested by hand and cured in bulk curing barns with the standard three-phase curing process (including yellowing phase, leaf drying phase and stem drying phase). A total of 35 flue-cured tobacco samples (ranked as $\mathrm{C} 3 \mathrm{~F}$ ) were collected after the curing period from three districts, including Liangshan, Guangyuan and Yibin. They are located in the south-west, north and south area of Sichuan Province, respectively, which have different climatic conditions. Sampling was carried out during October and November in 2011. The sample group was composed of six tobacco cultivars, including Yunyan 85, Yunyan 87, Yunyan 97, Honghuadajinyuan, Zhongyan 103 and K326. All information about samples is displayed in Table-1.

Sample preparation: Tobacco leaves were dried at $40^{\circ} \mathrm{C}$ for $6 \mathrm{~h}$ and ground into powder. Volatile compounds were extracted with simultaneous distillation-solvent extraction (SDE) apparatus. For each extraction, $20 \mathrm{~g}$ of tobacco sample, $10 \mathrm{~g}$ sodium sulphate and $300 \mathrm{~mL}$ ultra-pure water were placed in a $1 \mathrm{~L}$ flask and heated with a boiling water bath, $50 \mathrm{~mL}$ dichloromethane was added to a $100 \mathrm{~mL}$ flask heated with 40 ${ }^{\circ} \mathrm{C}$ water bath and the temperature of the circulating cooling water system was operated at $8{ }^{\circ} \mathrm{C}$. Steam distillation was stopped after $2 \mathrm{~h}$, while solvent extraction was continued for further $15 \mathrm{~min}$. The extract was concentrated to $1 \mathrm{~mL}$ at $10^{\circ} \mathrm{C}$ using nitrogen-purge apparatus. The concentrated solution was dehydrated with anhydrous sodium sulphate for at least $12 \mathrm{~h}$, of which $2 \mu \mathrm{L}$ was injected into the GC-MS system for analysis.
GC-MS conditions: The auto system Shimaszu QP 2010 GC-MS was employed for the analysis of volatile components. Low-bleed GC-MS column Rtx-5Ms, (5 \% diphenyl/95\% dimethyl polysiloxane, $30 \mathrm{~m} \times 0.25 \mathrm{~mm}$ ID $\times 0.25 \mu \mathrm{m}$ as used to resolve the volatiles. In terms of GC temperature programming, the oven temperature was set at $50{ }^{\circ} \mathrm{C}$ and kept there for $2 \mathrm{~min}$, then raised to $110^{\circ} \mathrm{C}$ at a ramp of $8^{\circ} \mathrm{C} / \mathrm{min}$ and kept there for $2 \mathrm{~min}$, then raised to $150^{\circ} \mathrm{C}$ at of $3{ }^{\circ} \mathrm{C} / \mathrm{min}$ and kept there for $2 \mathrm{~min}$, then raised to $200^{\circ} \mathrm{C}$ at of $5^{\circ} \mathrm{C} / \mathrm{min}$ and kept there for $5 \mathrm{~min}$ and finally raised to $240{ }^{\circ} \mathrm{C}$ at $10^{\circ} \mathrm{C} / \mathrm{min}$ and kept there for $2 \mathrm{~min}$. The carrier gas was helium. Mass spectrometry was operated at $230{ }^{\circ} \mathrm{C}$ in electron impact mode $(70$ $\mathrm{eV})$, scanning from $\mathrm{m} / \mathrm{z} 40-600$ in $0.3 \mathrm{~s}$ with an $0.2 \mathrm{~s}$ scanning interval time, the temperature of the GC-MS interface was $250{ }^{\circ} \mathrm{C}$ and the voltage of the photoelectric multiplier tube (PMT) was $200 \mathrm{~V}$.

Validation parameters for the GC-MS method: The linearity and sensitivity of the method were investigated using available reference standard phenylethyl acetate, with concentrations ranging from 5 to $200 \mu \mathrm{g} \mathrm{mL} / \mathrm{L}$. Each point on the calibration curve, expressed as peak area, was obtained from a minimum of three replicates of measurements (RSD < 0.02). The relationship between the peak area and standard concentration was determined by linear regression with $\mathrm{R}^{2}>0.99$.

Semi-quantitative and qualitative of volatile: Data analysis was performed using GCMS solution (Shimadzu, JAP). After peak smoothing and aligning of GC spectra, the quantification of the internal standard was done manually for each sample, then the peak area was corrected with reference to the internal standard. Semi-quantitative analysis was based on the peak area percentage method using GC-MS total ion chromatograms. Peak area percentage was calculated by comparing each peak area to the total peak area in the same sample, without considering calibration factors. GC peaks with relative peak area exceeding $0.1 \%$ were extracted. Identification of selected peaks was based on the NIST05.L MS library (National Institute of Standards and Technology, Gaithersburg, USA).

Statistical analysis: Comparison of the means was achieved using a one-way analysis of variance (ANOVA) using the SPSS 17.0 statistics software (SPSS Inc.). Data was centered and pareto scaled after importing to SIMCA software. Principal component analysis (PCA) and partial least

\begin{tabular}{|c|c|c|c|c|c|c|c|c|}
\hline \multicolumn{9}{|c|}{$\begin{array}{c}\text { TABLE-1 } \\
\text { TOBACCO SAMPLES FROM SICHUAN PROVINCE }\end{array}$} \\
\hline No. & Geographical origin & Cultivar & No. & Geographical origin & Cultivar & No. & Geographical origin & Cultivar \\
\hline 1 & LS & Y87 & 13 & YB & Y97 & 25 & GY & Y85 \\
\hline 2 & LS & Y85 & 14 & YB & K326 & 26 & GY & Y85 \\
\hline 3 & LS & Y85 & 15 & YB & $\mathrm{HD}$ & 27 & GY & Y85 \\
\hline 4 & LS & Y85 & 16 & YB & K326 & 28 & GY & Y85 \\
\hline 5 & LS & Y87 & 17 & YB & HD & 29 & GY & Y85 \\
\hline 6 & LS & Y85 & 18 & YB & HD & 30 & GY & Y85 \\
\hline 7 & LS & Y87 & 19 & YB & Z103 & 31 & GY & Y87 \\
\hline 8 & LS & Y87 & 20 & YB & Z103 & 32 & GY & Y87 \\
\hline 9 & LS & Y85 & 21 & YB & Y97 & 33 & GY & Y87 \\
\hline 10 & LS & Y87 & 22 & YB & K326 & 34 & GY & Y87 \\
\hline 11 & LS & Y85 & 23 & YB & Z103 & 35 & GY & Y87 \\
\hline 12 & LS & Y87 & 24 & YB & K326 & - & - & - \\
\hline
\end{tabular}

Origin: LS-Liangshan district, YB-Yibin district, GY- Guangyuan district Tobacco Cultivar: Y85-Yunyan85, Y87-Yunyan87, Y97-Yunyan97, HDHonghuadajinyuan, Z103-Zhongyan103, K326. 
squares discrimination analysis ( PLS-DA) were performed with SIMCA-P version 13. PCA was used to overview data clustering trends and to identify outliers with Ellipse, Hotelling's T2 (95 \%). PLS-DA was carried out to reveal the relationship between samples and variables (tobacco volatiles). The leave out cross validation method was used to test the prediction classification ability.

\section{RESULTS AND DISCUSSION}

A total of 56 volatile compounds were detected with GCMS and identified (Table-2) by matching with NIST05.L MS library (similarity ratio $>80 \%$ ). The volatile compounds covered on average $95.46 \%$ of the total peak area recovered from the GC spectra. Volatile compounds were semi-quantified by means of peak area percentage (not shown).

Geographical discrimination of flue-cured tobacco using volatile compound analysis: Principal component analysis (PCA) was used to provide an overview of the capacity of the variables (volatile compounds) to discriminate tobacco samples from different regions. All data derived from 56 volatile compounds of 35 samples were subjected to a PCA-class model with geographical origins taken as class ID. In referencing three independent PCA score plots (not shown) for each sample group from different regions, no samples were identified as possible outliers. After applying PCA to the raw data set of 35 samples, four PCs were extracted according to the NIPALS algorithm $(\mathrm{R} 2 \mathrm{X}=0.942, \mathrm{Q} 2=0.547)$. The percentage of variance explained by each $\mathrm{PC}$ was 46.5, 29.5, 11.5 and $6 \%$, respectively. The PCA-X score scatter plot (not shown) reveals that they could be classified into three clusters coincident to the three sampled geographical origins.

Supervised PLS-DA was carried out to reveal the relationship between volatile compounds and the three clusters. The initial PLS-DA model was calculated using all 56 volatile compounds. The R2X and Q2X values of the PLS-DA models described the model quality, R2X indicates how well the model fits and Q2X indicates the model predictability. The first PLSDA model was established using two components and revealed RX2 (cum), RY2 (cum) and Q2 (cum) values of 0.80, 0.62 and 0.54 , respectively. The score plots of PLS-DA show correlation of samples and the weights scatter plot of PLS-DA reveal the relationship between variables and their loading. The PLS-DA score plot (Fig. 1a) also displays a clear separating trend in the three classes, according to the sample origins as well as the PCA-X score scatter plot. However, the PLS-DA loading plot (Fig. 1b) failed to show the correlation between variables and sample groups clearly because of the serious overlap of most variables. To decrease overlap and to improve the interoperating ability of the PLS-DA loading plot, unimportant variables for the PLS-DA were reduced. Variable importance for the projection (VIP) plot of the PLS-DA (Fig. 2) displayed the contribution value of each variable and using the VIP > 1, nine variables were judged as being important.

PLS-DA was performed again with the nine variables (far left in Fig. 2) with VIP > 1. In the PLS-DA loading plot, Xvariables situated in the vicinity of the dummy Y-variables have the highest discriminatory power between the classes. Based on the loading (Fig. 1d) and score plots (Fig. 1c) of the

\begin{tabular}{|c|c|c|c|c|c|}
\hline \multicolumn{6}{|c|}{$\begin{array}{l}\text { TABLE- } 2 \\
\text { MPOUNDS IN FLUE-CURED TOBACCO FROM SICHUAN }\end{array}$} \\
\hline No. & Compound & $\mathrm{RT}$ & No. & Compound & RT \\
\hline 1 & 2-Butylfuran & 6.446 & 29 & Cedrol & 26.880 \\
\hline 2 & 2,3-Dihydro-benzofuran & 7.251 & 30 & Megastigmatrienone $\mathrm{C}$ & 27.084 \\
\hline 3 & 6-Methyl-2-heptanone & 8.326 & 31 & Megastigmatrienone D & 27.437 \\
\hline 4 & Benzyl alcohol & 10.471 & 32 & 4-(3-Hydroxy-1-butenyl)-3,5,5-trimethyl-2-cyclohexen-1-one & 27.958 \\
\hline 5 & Benzeneacetaldehyde & 10.776 & 33 & Heneicosane & 28.877 \\
\hline 6 & Acetophenone & 11.410 & 34 & Heptadecane & 29.272 \\
\hline 7 & Nonanal & 12.342 & 35 & Norphytane & 29.432 \\
\hline 8 & Phenylethyl alcohol & 12.645 & 36 & Palmitaldehyde & 29.765 \\
\hline 9 & 2,6,6-Trimethyl-2-cyclohexene-1,4-dione & 13.481 & 37 & Hexa-hydrofarnesol & 30.155 \\
\hline 10 & Nonanoic acid & 16.434 & 38 & Tetradecanoic acid & 30.990 \\
\hline 11 & Indole & 17.360 & 39 & Isopulegyl acetate & 31.230 \\
\hline 12 & 4-Ethenyl-2-methoxy phenol & 17.850 & 40 & Anthracene & 31.930 \\
\hline 13 & Farnesol & 17.992 & 41 & Phytane & 32.336 \\
\hline 14 & Hexahydrofarnesol & 18.783 & 42 & Neophytadiene & 33.305 \\
\hline 15 & Nicotine & 18.865 & 43 & Hexahydrofarnesyl acetone & 33.431 \\
\hline 16 & Solanone & 19.289 & 44 & 3,7,11,15-Tetramethyl-2-hexadecen-1-ol & 33.860 \\
\hline 17 & Hexahydropseudoionone & 20.412 & 45 & 3,7,11-Trimethyl-2,10-dodecadien-1-ol & 34.179 \\
\hline 18 & Myosmine & 21.276 & 46 & Farnesyl acetone & 35.518 \\
\hline 19 & Geranyl acetone & 21.950 & 47 & Palmitic acid, methyl ester & 35.632 \\
\hline 20 & Nicotine-N'-oxide & 22.859 & 48 & 3,7,11,15-Tetramethyl-2-hexadecene & 35.856 \\
\hline 21 & 2,4-bis(1,1-Dimethylethyl) phenol & 23.776 & 49 & Hexadecanoic acid & 36.718 \\
\hline 22 & 6-Methoxy-3-methylbenzofuran & 24.171 & 50 & 3-(4,8,12-Trimethyltridecyl)furan & 37.037 \\
\hline 23 & Dihydroactinidiolide & 24.679 & 51 & 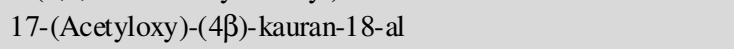 & 41.430 \\
\hline 24 & Megastigmatrienone A & 25.501 & 52 & $\beta-4,8,13$-Duvatriene-1,3-diol & 42.095 \\
\hline 25 & Megastigmatrienone $\mathrm{B}$ & 26.018 & 53 & Phytol & 43.090 \\
\hline 26 & Hexadecane & 26.322 & 54 & $\alpha-4,8,13$-Duvatriene-1,3-diol & 43.465 \\
\hline 27 & Diethyl Phthalate & 26.416 & 55 & Linolenic acid, methyl ester & 44.503 \\
\hline 28 & Myristaldehyde & 26.789 & 56 & Phthalic acid, dioctyl ester & 64.193 \\
\hline
\end{tabular}




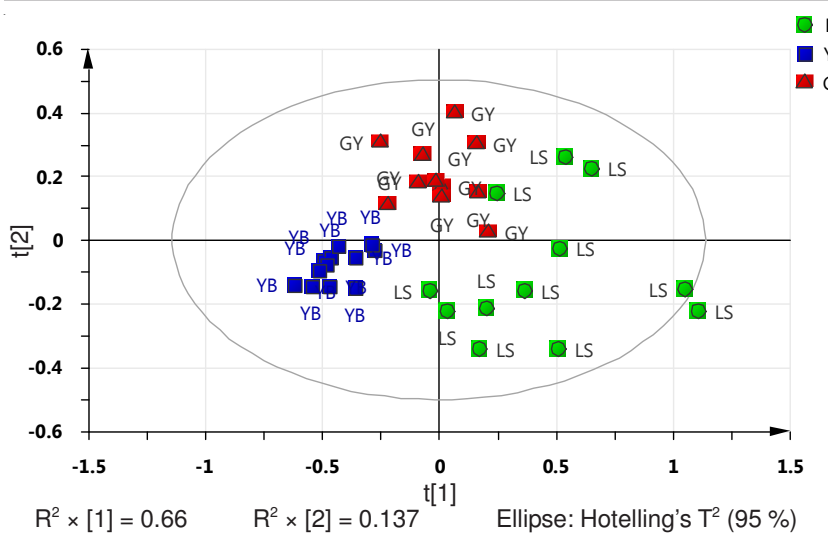

(a)

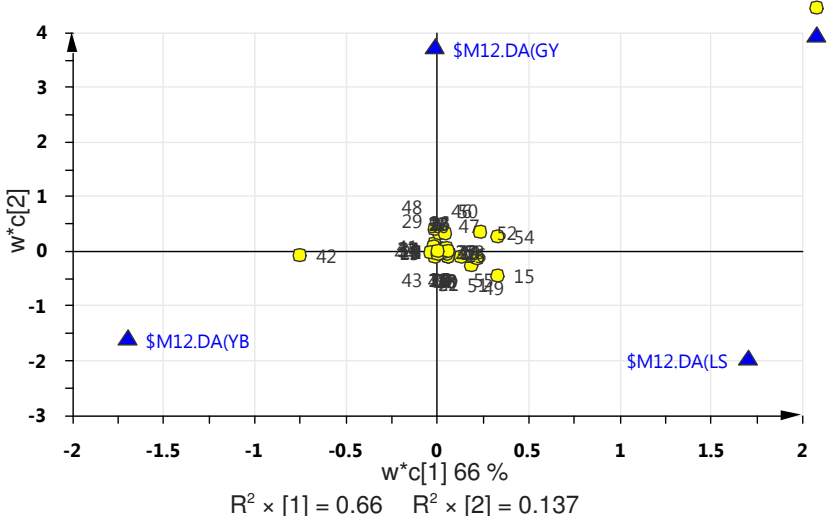

(b)

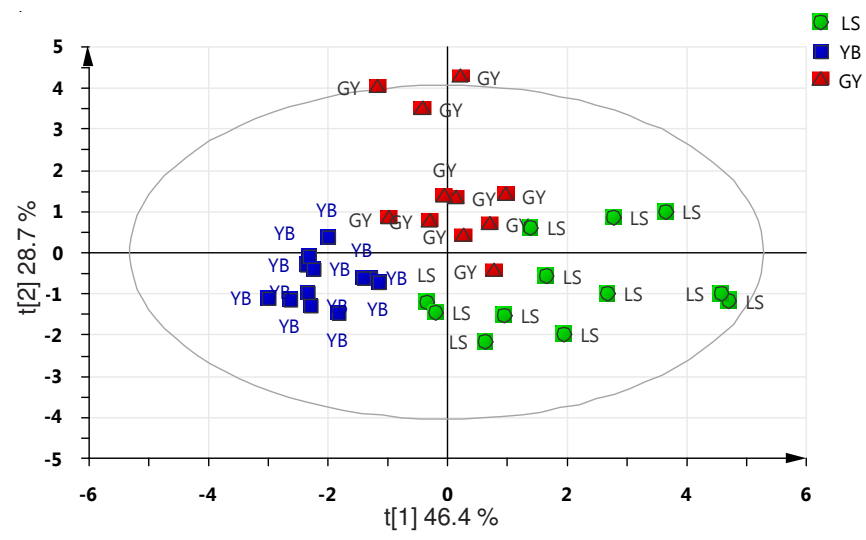

(c)

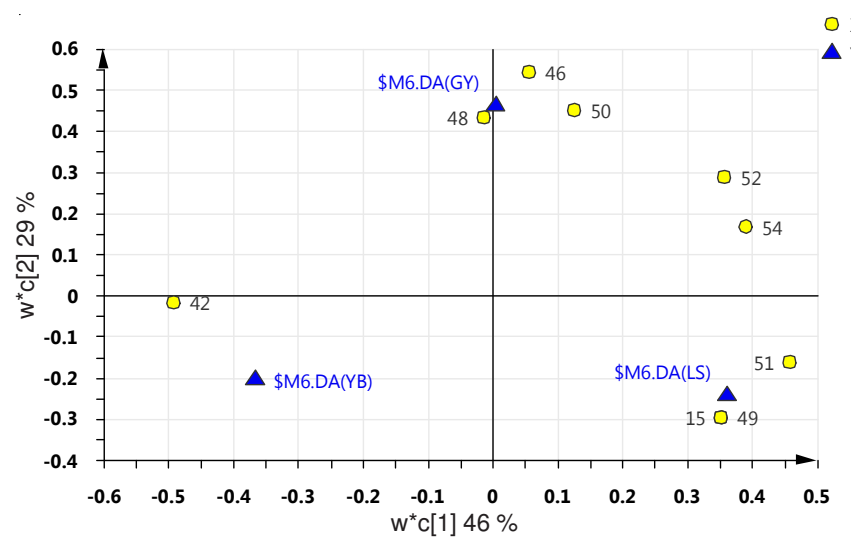

(d)

Fig. 1. PLS-DA score plot and loading plot

second PLS-DA, it is easy to find that Var 42 is the most important variable to discriminate YB samples; Var 48, Var 46 and Var 50 are the most important three variables to discriminate GY samples; Var 15, Var 49, Var 51, Var 52 and Var 54 are the most important variables for discriminating LS samples.

Contribution scores and the analysis of variance (ANOVA) of the variables of the second PLS-DA are shown in Table-3, displayed on the left and right sides, respectively. The contribution scores of PLS-DA show the weighted difference between certain groups and the average of three groups. It also indicates which variables in a group deviate most from the group average. The signs of contribution scores (positive $=$ higher and negative=lower content than average) indicate in which direction the variables deviate. ANOVA highlighted statistically significant differences $(p<0.05)$ of the nine variables among the sample groups produced in three geographical areas. This can explain why they are so important in discriminating geographical origin.

Validity and reliability of the second PLS-DA models were tested by permutation tests and analysis of variance testing of cross-validated predictive residuals (CV-ANOVA). Permutation tests were performed with 20 random reclassifications. The permutation plot displays the correlation coefficient between the original $y$-variable and the permuted $y$-variable on the $\mathrm{X}$-axis versus the cumulative $\mathrm{R} 2$ and $\mathrm{Q} 2$ on the $\mathrm{Y}$-axis and draws the regression line. The criteria for validity of PLS-DA models

\begin{tabular}{|c|c|c|c|c|c|c|c|c|c|}
\hline \multicolumn{10}{|c|}{ TABLE-3 } \\
\hline \multirow{3}{*}{ Var ID } & \multicolumn{3}{|c|}{ Contribution } & \multicolumn{6}{|c|}{ ANOVA } \\
\hline & \multirow{2}{*}{ LS } & \multirow{2}{*}{ YB } & \multirow{2}{*}{ GY } & \multicolumn{2}{|c|}{$\mathrm{LS}$} & \multicolumn{2}{|c|}{ YB } & \multicolumn{2}{|c|}{ GY } \\
\hline & & & & Mean & SD & Mean & SD & Mean & SD \\
\hline 15 & $0.9601^{*}$ & -0.5342 & -0.4077 & $3.70 \mathrm{a}$ & 2.92 & $0.29 b$ & 0.29 & $0.43 b$ & 0.64 \\
\hline 42 & $-1.2915^{*}$ & $1.4103^{*}$ & -0.0036 & $79.41 \mathrm{c}$ & 5.17 & $91.82 \mathrm{a}$ & 1.37 & $85.07 \mathrm{~b}$ & 1.96 \\
\hline 46 & -0.2268 & -0.3437 & $1.4474^{*}$ & $0.23 \mathrm{a}$ & 0.04 & $0.14 b$ & 0.04 & $0.22 \mathrm{a}$ & 0.06 \\
\hline 48 & -0.2120 & -0.1503 & $0.9157^{*}$ & $0.43 b$ & 0.20 & $0.48 b$ & 0.33 & $1.20 \mathrm{a}$ & 1.09 \\
\hline 49 & $0.9658^{*}$ & -0.5373 & -0.4114 & $1.16 \mathrm{a}$ & 0.97 & $0.05 \mathrm{~b}$ & 0.05 & $0.09 \mathrm{~b}$ & 0.07 \\
\hline 50 & -0.0619 & -0.3796 & $1.0058^{*}$ & $0.61 b$ & 0.19 & $0.391 b$ & 0.16 & $0.97 \mathrm{a}$ & 0.61 \\
\hline 51 & $1.3004^{*}$ & $-1.0635^{*}$ & -0.1128 & $2.21 \mathrm{a}$ & 0.53 & $1.01 \mathrm{c}$ & 0.26 & $1.39 \mathrm{~b}$ & 0.30 \\
\hline 52 & 0.5222 & $-0.9935^{*}$ & 0.4226 & $2.69 \mathrm{a}$ & 1.15 & $0.96 b$ & 0.48 & $2.67 \mathrm{a}$ & 0.87 \\
\hline 54 & 0.7085 & $-1.0212^{*}$ & 0.1484 & $4.53 \mathrm{a}$ & 2.04 & $1.49 \mathrm{~b}$ & 0.65 & $3.82 \mathrm{a}$ & 1.10 \\
\hline
\end{tabular}




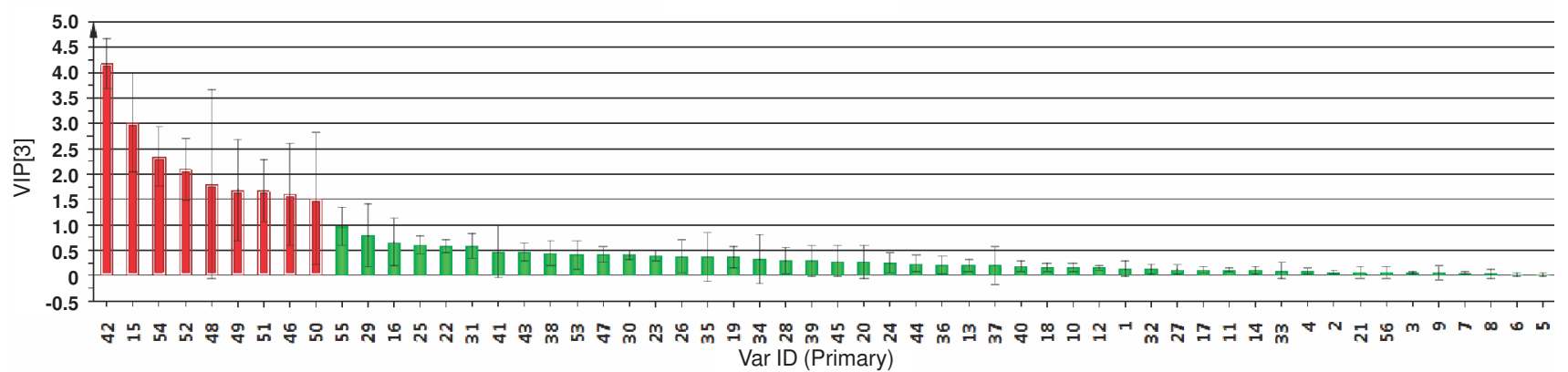

Fig. 2. VIP analysis on 56 variables of the first PLS-DA. Note: column marked in red represents variables with variable influence of projection (VIP) values exceeding 1

are all blue, Q2-values to the left are lower than the original points to the right or the blue regression line of the Q2-points intersect the vertical axis (on the left) below zero. Fig. 3 indicates that all of the second PLS-DA models with nine variables have good fitness and predictability. Analysis of variance of sevenfold cross-validation predictive residual (CV-ANOVA) show the $\mathrm{P}=1.84612 \mathrm{e}-006<0.05$. The misclassification test (Table4) indicates that the second PLS-DA can discriminate tobacco samples from the three districts with a correct classification rate of $91.43 \%$. This supports the view that environmental conditions (climate, rainfall, soil quality) and culture technology are closely related to geographical origin, which directly affect the character of volatiles ${ }^{31}$.

TABLE-4

MISCLASSIFICATION TEST OF THE SECOND PLS-DA

\begin{tabular}{cccccc}
\hline \multirow{2}{*}{ Origin } & $\begin{array}{c}\text { Sample } \\
\text { members }\end{array}$ & \multicolumn{4}{c}{ Result } \\
\cline { 3 - 6 } & Correct $(\%)$ & LS & YB & GY \\
\hline LS & 12 & 83.33 & 10 & 2 & 0 \\
YB & 12 & 100 & 0 & 12 & 0 \\
GY & 11 & 90.91 & 1 & 0 & 10 \\
Total & 35 & 91.43 & 11 & 14 & 10 \\
\hline
\end{tabular}

Note: Fishers prob. $=3.1 \mathrm{e}-012$.

Variety discrimination of flue-cured tobacco using volatile compound analysis: In this study, all samples belong to six varieties, respectively, with the sample numbers ranging from 2 to 11 . The score plot of PCA to determine primary observation of the six varieties failed to reveal separation between varieties (Fig. 4). Moreover, the PLS-DA model could not be established with SIMCA due to the fact that no significant principle component could be extracted. Considering that samples from Liangshan and Guangyuan are very suitable for analyzing the difference in volatiles between $\mathrm{Y} 85$ and $\mathrm{Y} 87$, further attempts to detect the difference between Y 85 and Y 87 samples on volatiles by OPLS-DA was carried out, because both of them have six samples of Y85 and five or six samples of Y87. However, no predictive components could be extracted from the Liangshan and Guangyuan samples. Therefore, no volatile could be identified as a useful characteristic to discriminate tobacco varieties in this study.

\section{Conclusion}

Volatile profiling based on GCMS spectra was used to analyze the differences among tobacco samples extracted from three districts with the aim of finding markers useful for identifying geographical origin. The results demonstrate that a

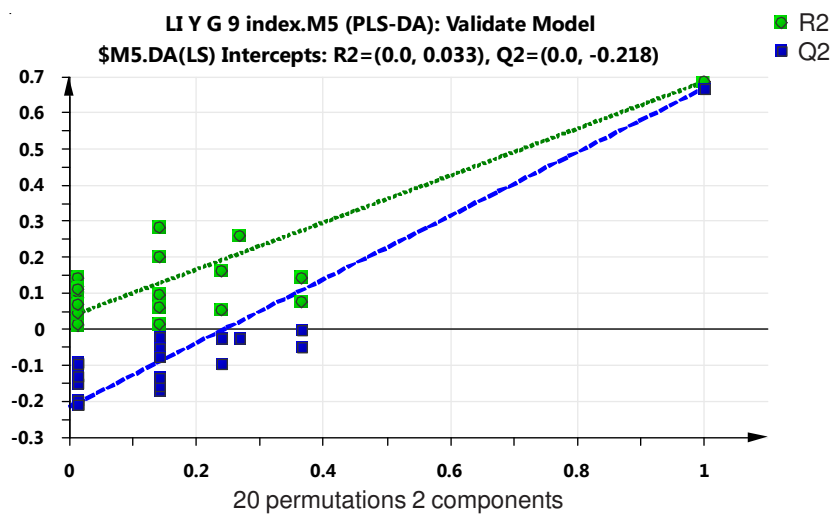

(a)

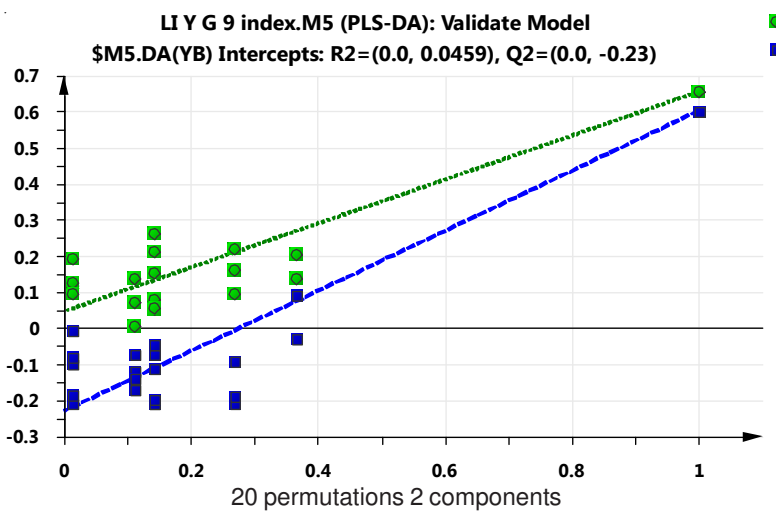

(b)

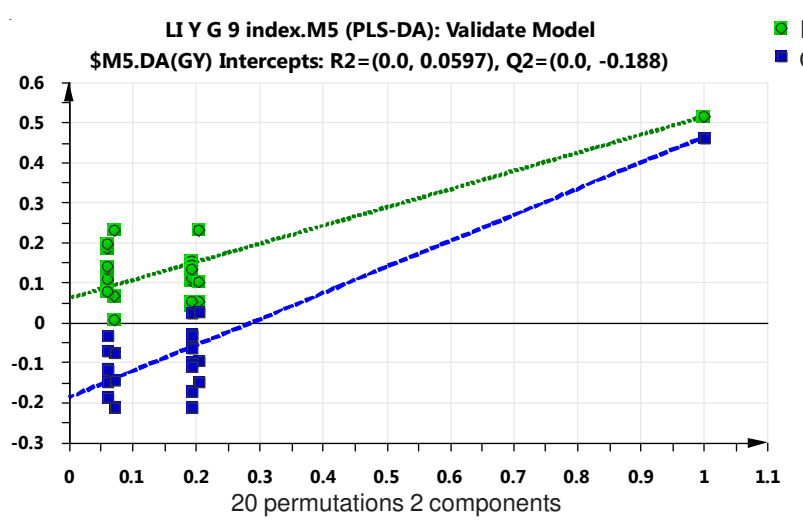

(c)

Fig. 3. Results from response permutation test of PLS-DA models. The vertical axis gives the R2 (green) and Q2 (blue) values of the original model (far right) and the Y-permuted models further to the left. The horizontal axis shows the correlation between the permuted $y-$ vectors and the original y-vector for the selected. (a): LS, (b): YB, (c): GY 


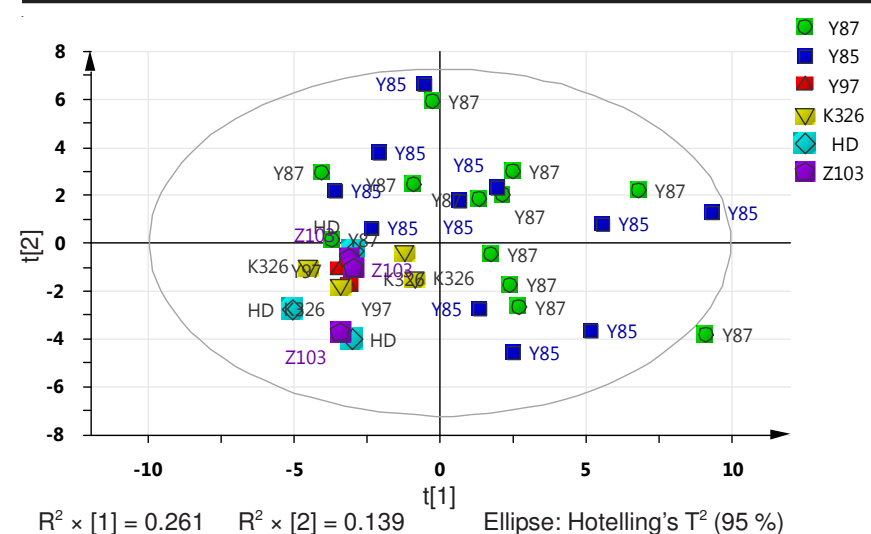

Fig. 4. PCA-X score plot of six tobacco varieties

combination of GCMS and PLS-DA multivariate analyses allows comparisons of overall volatile fingerprints and that this technique can be applied to identify differences between tobacco samples.

Distinct separations between tobacco samples from three districts were observed in chemometric analyses using PCA and PLS-DA. Nine volatile components were selected as candidate biomarkers that could be used to quickly and easily differentiate tobacco samples. However, no volatile component was selected as a biomarker to identify tobacco varieties in this study. In summary, this study demonstrates that GCMSbased volatile fingerprinting is a useful tool for distinguishing origins of tobacco samples, coupled with multivariate statistical analysis. The reasons for the differences in volatile profiles in tobacco leaves sampled from different geographical origins are not fully understood, and further investigations are needed.

\section{REFERENCES}

1. X.Y. Zhang, X.S. Su and B.N. Jiao, Food Sci., 31, 271 (2010)

2. K.A. Anderson, B.A. Magnuson, M.L. Tschirgi and B. Smith, J. Agric. Food Chem., 47, 1568 (1999).

3. M.J. Latorre, R. Pena, S. Garcia and C. Herrero, Analyst, 125, 307 (2000).
4. F. Di Giacomo, A. Del Signore and M. Giaccio, J. Agric. Food Chem., 55, 860 (2007).

5. X.Y. Chang, S.M. Fu, H. Liu, N. Chen, X.F. Zhao, B.Q. Zhu and X.L. Tu, Chin. J. Geochem., 30, 138 (2011).

6. F. Longobardi, G. Casiello, D. Sacco, L. Tedone and A. Sacco, Food Chem., 124, 1708 (2011).

7. S. Wilkinson, A.L. Archibald, C.S. Haley, H.J. Megens, R.P. Crooijmans, M.A. Groenen, P. Wiener and R. Ogden, BMC Genomics, 13, 580 (2012).

8. S.K. Wasser, W.J. Clark, O. Drori, E.S. Kisamo E, C. Mailand, B. Mutayoba and M. Stephens M., Conserv. Biol., 22, 1065 (2008).

9. P. Zunin, R. Boggia, P. Salvadeo and F. Evangelisti, J. Chromatogr. A, 1089, 243 (2005).

10. S. Risticevic, E. Carasek and J. Pawliszyn, Anal. Chim. Acta, 617, 72 (2008).

11. X.Y. Zhang, X.S. SU and B.N. Jiao, Food Sci., 31, 271 (2010).

12. J. Reinhard, M.V. Srivivasan and S. Zhang, Nature, 427, 411 (2004).

13. T.D. Sharkey and S. Yeh, Ann. Rev. Plant Physiol., 52, 407 (2001).

14. C.M. De Moraes, M.C. Mescheer and J.H. Tumlinson, Nature, 410, 577 (2001).

15. A. Kessler and I.T. Baldwin, Science, 291, 2141 (2001).

16. B. Toso, G. Procida and B. Stefanon, J. Dairy Res., 69, 569 (2002).

17. R.A. Shellie, S.D.H. Poynter and J.F. Li, J. Sep. Sci., 32, 3720 (2009).

18. J.S. Camara, M.A. Alves and J.C. Marques, Talanta, 68, 1512 (2006).

19. M. Staudt and N. Bertin, Plant Cell Environ., 21, 385 (1998).

20. J. Gershenzon, M.E. McConkey and R.B. Croteau, Plant Physiol., 122, 205 (2000).

21. L. Mondello, R. Costa and P.Q. Tranchida, J. Sep. Sci., 28, 1101 (2005).

22. T. Cajka, J. Hajslova, F. Pudil and K. Riddellova, J. Chromatogr. A, 1216, 1458 (2009).

23. I. Stanimirova, B. Ustun. T. Cajka, K. Riddelova, J. Hajslova, L.M.C. Buydens and B. Walczak, Food Chem., 118, 171 (2010).

24. N. Araghipour, J. Colineau, A. Koot, W. Akkermans, J.M.M. Rojas, J. Beauchamp, A. Wisthaler, T.D. Märk, G. Downey, C. Guillou, L. Mannina and S. van Ruth, Food Chem., 108, 374 (2008).

25. N.S. Ye, L. Zhang and X. Gu, Food Anal. Methods, 5, 856 (2012).

26. L. Pillonel, S. Ampuero, R. Tabacchi and J. Bosset, Eur. Food Res. Technol., 216, 179 (2003).

27. J.B. Cai, B.Z. Liu, P. Ling and Q.D. Su, J. Chromatogr. A, 947, 267 (2002).

28. J.L. Shi, H.Q. Yang and C.M. Song, J. Yunnan Agric.Univ., 26, 790 (2011).

29. S.K. Wang, H.Y. Luo and Y. Wang, Chin. Tobac. Sci., 32, 10 (2011).

30. X.L. Zhu, J. Yang and Y.B. Xu, Acta Tobac. Sin., 12, 33 (2010).

31. E. Pouliarekou, A. Badeka, M. Tasioula-Margari, S. Kontakos, F. Longobardi and M.G. Kontominas, J. Chromatogr. A, 1218, 7534 (2011). 